\title{
Massive degenerated leiomyomas masquerading ovarian neoplasm
}

\author{
Deeksha Pandy ${ }^{1}$, P Priyadarshini ${ }^{1}$, Mirajkar S Feroz ${ }^{1}$, P S Roopa ${ }^{1}$, Ranjini Kudva ${ }^{1}$, Jyothi Shetty ${ }^{1}$, \\ Vani Ramkumar ${ }^{2}$
}

Sri Lanka Journal of Obstetrics and Gynaecology 2011; 33: 163-165

Key words: leiomyoma uteri, degeneration, ovarian tumor.

\section{Introduction}

Leiomyoma uteri are the most common tumors of the uterus and female pelvis ${ }^{1}$. Common presentations include menorrhagia and dysmenorrhea. The diagnosis is straightforward and is confirmed by ultrasonography. Recently we encountered two cases of massive cystic degeneration of leiomyomata, mimicking ovarian malignancy that confused both the clinician and radiologist.

Case 1: A 46-year-old, multiparous lady was referred to our tertiary care centre, with the diagnosis of ovarian malignancy. She had noticed abdominal distension for a month and felt dull aching pain in abdomen for one week. There was a history of reduced appetite, early satiety, and frequency of micturition. She had a BMI of $25.8 \mathrm{~kg} / \mathrm{m}^{2}$, mild pallor and no lymphadenopathy. Abdominal examination revealed a cystic mass corresponding to 32 weeks size of gravid uterus. On pelvic examination cervix was hitched behind the pubic symphysis, cystic mass was occupying both the lateral and posterior vaginal fornices. Uterus could not be made out separately. Sonogram showed large $25 \times 20 \mathrm{~cm}$ well defined cystic lesion arising from the pelvis extending up to the epigastric area with multiple thick septations showing internal vascularity. Doppler studies of the uterus was normal. However, both ovaries could not be identified separately. There was evidence of bilateral hydronephrosis. CA 125 was not much elevated $(82 \mu \mathrm{g} / \mathrm{ml})$. Clinical findings as well as sonography suggested the diagnosis of ovarian tumor.

At laparotomy, a benign looking huge cystic mass was visualized. It was carefully punctured with a trocar and decompressed for the sake of easy removal. Around three liters of clear serous fluid was

\footnotetext{
${ }^{1}$ Kasturba Medical College Manipal, India.

${ }^{2}$ Melaka Manipal Medical College Manipal, India.

Correspondence: Deeksha Pandy

E-mail: deekshiiiobg@gmail.com
}

aspirated, by means of 3-4 separate punctures. On delivering the decompressed cyst out of the abdomen, the pedicle was traced. Surprisingly the pedicle was found to be arising from the left cornua of the otherwise normal uterus. The pedicle was clamped, cut and ligated (Figure 1). Surgery was completed by performing total hysterectomy with bilateral salpingooophorectomy.

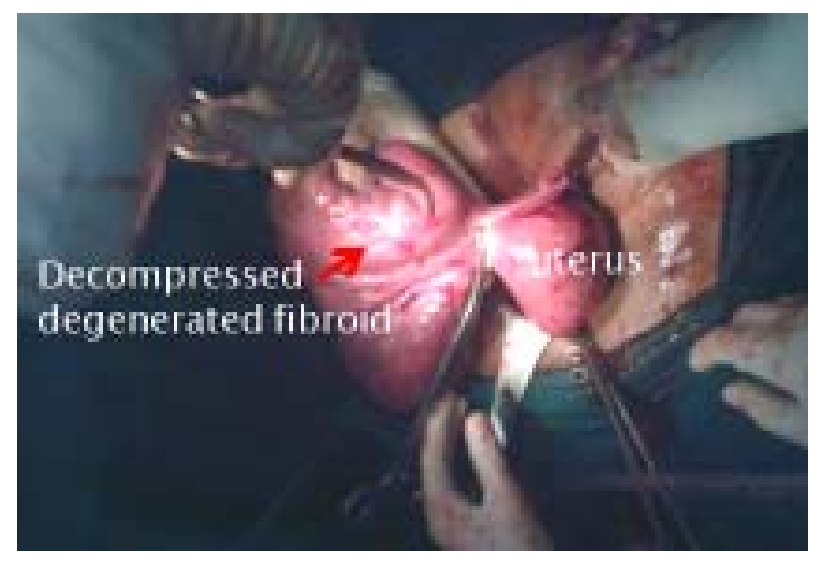

Figure 1. Intraoperative picture showing the origin of huge degenerated fibroid from uterus.

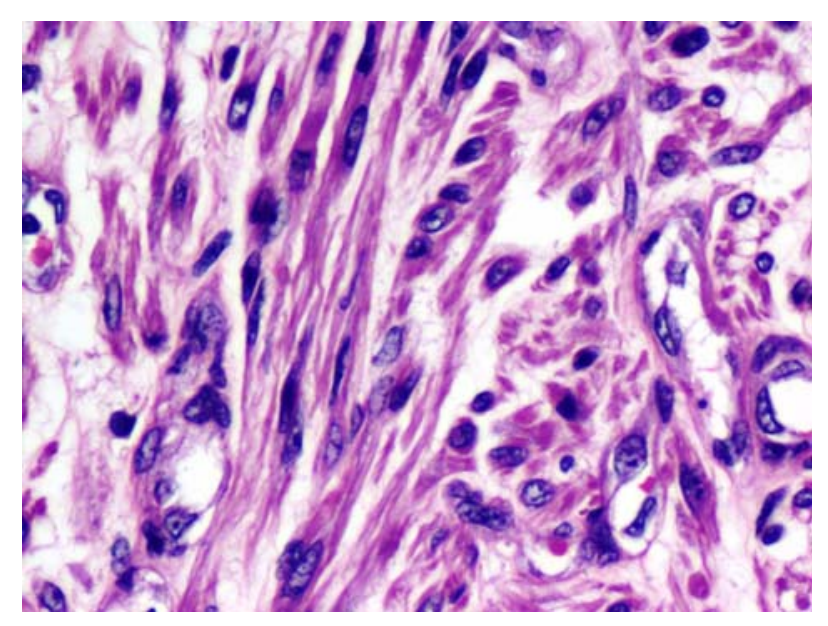

Figure 2. Histopathologic section H \& E $\times 200$ showing cells with uniform cigar shaped nuclei in interlacing bundles. 
Gross examination revealed a cystic mass weighing $1.12 \mathrm{~kg}$ and measuring $23 \times 14 \times 3 \mathrm{~cm}$. Cut section showed multiloculated cyst filled with serous fluid along with a solid area measuring $11 \times 9 \times 3 \mathrm{~cm}$. Cut section of this solid area showed firm grey white areas along with tiny cystic spaces and focal hemorrhagic areas. Microscopic sections revealed a neoplasm composed of benign spindle shaped cells with cigar shaped nuclei arranged in fascicles with interspersed mast cells, thick walled blood vessels, edema, hyalinization and extensive cystic degeneration (Figure 2). Thus the final diagnosis was subserous leiomyoma with extensive cystic degeneration.

Case 2: A 48-year-old multiparous lady presented with complaints of giddiness and easy fatigability for one month. Her cycles were regular with normal flow except for the last menstruation, around a month ago that was heavy and lasted for around 10 days. On examination she was cachexic with BMI of 16.46 $\mathrm{kg} / \mathrm{m}^{2}$. She had severe pallor. Per abdominally a mobile mass of variegated consistency was palpable which corresponded to a 24 weeks size gravid uterus. There was no ascites. On bimanual pelvic examination the same mass was felt. Ultrasonography showed a heterogenous $15 \times 12 \mathrm{~cm}$ mass with cystic and solid components and thick septations, suggestive of ovarian malignancy (Figure 3). The hemoglobin was $2 \mathrm{~g} / \mathrm{dl}$ and she was transfused with five pints of packed cells preoperatively. CA125 was within normal limits $(18 \mu \mathrm{g} / \mathrm{ml})$.

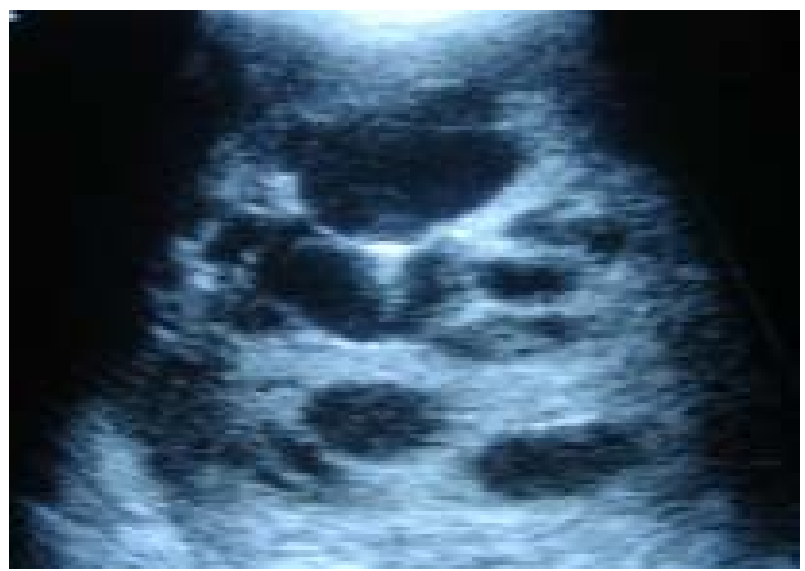

Figure 3. Intraoperative picture showing the origin of huge degenerated fibroid from uterus.

Intraoperatively to our surprise the uterus was uniformly enlarged to $20 \times 15 \mathrm{~cm}$ and adnexae were normal. On cut section the uterus had multiple cavities filled with cheesy and gelatinous material (Figure 4). Histopathological examination revealed that it was cystic degeneration of large submucous leiomyoma.

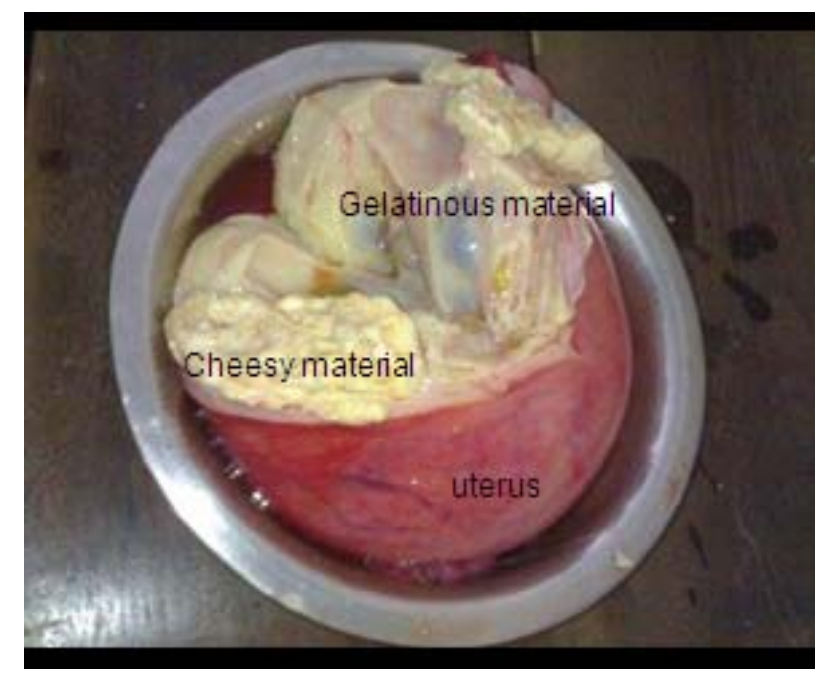

Figure 4. Cut section of uterus showing multiple cavities filled with cheesy and gelatinous material.

\section{Discussion}

Fibroids are the most common benign neoplasms of the uterus. Usually the diagnosis is straightforward. At times however, it might prove to be a diagnostic dilemma, mostly if associated with secondary changes. The most common secondary change in leiomyoma is hyaline degeneration.

Eventually these leiomyomas may become liquefied and form cystic cavities filled with clear liquid or gelatinous material. Sometimes the cystic change is so great that the leiomyoma becomes a mere shell and is truly a cystic tumor ${ }^{1}$. To add on to the diagnostic conundrum sometimes the pedunculated degenerated fibroid might start wandering, deriving its blood supply from the intestine ${ }^{2}$. As in our second case not only subserous, but extensively degenerated submucus myomas may also present as ovarian tumors.

Ultrasonography is the primary and usually sufficient modality for confirming the diagnosis of clinically suspected leiomyoma of the uterus. Huge degenerated leiomyomas can still pose diagnostic challenges. In these atypical cases even tomography can be misleading ${ }^{3}$. Magnetic resonance imaging may be helpful in reaching accurate diagnosis preoperatively ${ }^{4,5}$.

The lesson to be learnt is a large, degenerated, leiomyoma can have non-specific symptoms like ovarian tumor, and it may mimic ovarian tumor on examination as well as on sonography. A differential diagnosis of the common fibroid should always be kept in mind in absence of ascites and if CA125 values not correlating with the size of the tumor, while dealing with a suspected adnexal mass. 


\section{References}

1. John A. Rock, Howard W. Jones. Leiomyomata Uteri and Myomectomy. In: Te Linde's Operative Gynecology. 10th ed. Philadelphia: Wolters Kluwer Health/Lippincott Williams \& Wilkins; 2008.

2. Christina E Danez, Heather R Macdonald. Massive cystic degeneration of a pedunculated leiomyoma. Fertil Steril 2008; 90(4): 1180-1.
3. SCA Low, CL Chong. A Case of Cystic Leiomyoma Mimicking an Ovarian Malignancy. Ann Acad Med Singapore 2004; 33: 371-4.

4. CH Tok, SI Bux, SI Mohamed, BK Lim. Degenerated uterine fibroid mimicking hydrometra: fallacy in CT. Biomed Imaging Interv J 2006; 2(4):e 42.

5. ChhaviKaushik, Akhila Prasad, Yashvant Singh, BP Baruah. Case Series: Cystic degeneration in Uterine Leiomyomas. Indian J Radiol Imaging 2008; 18(1): 69-72. 